\title{
A Theory of Biochemical Organization, Metabolic Pathways, and Evolution
}

\author{
HAROLD J. MOROWITZ \\ Krasnow Institute for Advanced Study, George Mason University, Fairfax, Virginia 22030 USA
}

Received June 25, 1999; accepted June 28, 1999

\begin{abstract}
Biogenesis and evolution are viewed from the perspective of the universality of the metabolic chart with respect to primary metabolism and the phylogenetic specificity of secondary metabolism. This analysis is developed within the context of the evolution of the universal ancestor through hierarchical networks of chemical reactions. (C) 1999 John Wiley \& Sons, Inc.
\end{abstract}

\section{INTRODUCTION}

I he theme of this article is the examination of biological and prebiological evolution in terms of the universal present-day graph of metabolic pathways. Since the core features of metabolism date back to the universal ancestor some 3.9 billion years ago, this perspective looks for continuity between biogenesis and evolution and seeks for a deep logic of evolution within the reductionist roots of quantum mechanics, the periodic table, and the boundary conditions of the planet Earth. This is not to deny the existence of frozen accidents and random events at any stage, but is to look at the framework in which they happen. This infrastructure provides certain profound deterministic aspects to the process of biogenesis.

The constructs to be utilized are energy, mass, and structure (information, organization, complexity, etc.) We lack a universally agreed-on term or concept for the third feature, but it can be understood in a general way. Matter is not, in fact, either the structureless mass of hydrodynamics or the point mass of classical mechanics. Matter in the tempera- ture range of biological objects consists of atoms, atomic nuclei, and electrons. Atoms, of course, have an electronic structure imposed by allowable quantum mechanical states and the Pauli exclusion principle. As a result, each type of atom has unique properties that determine its interactions with like atoms and those of other types. All form in the size range of nanometers or less is dependent on the details of atomicity. Structural information is, thus, dependent on the differences among the atoms of the periodic table. In biochemistry, information is not a mathematical abstraction; it is associated with molecular structures. Information in this context cannot be isolated from the details of chemistry.

There has been and continues to be a confusion between Shannon information, which is a measure on a normalized probability distribution, and anecdotal uses of the word information. These are deep questions or as physicist Wajciech Zurek [1] points out, "The specter of information is haunting the sciences." Because the states of interest in biochemistry can be identified with atoms and molecules, this provides a starting point. 
One method of evaluating the structural complexity of an object is to determine the size of the shortest message that describes the object. This structural view can be applied to a snapshot of an object that is changing in time. Since living cells survive temperatures near absolute zero when there is nothing but structure, this snapshot point of view would appear to be valid in biochemistry. Consider the case of a homogeneous solution of molecules. The unique Simplified Molecular Input Line Entry System is a chemical language that represents any molecule by a linear sequence of symbols [2]. For example, D-alanine is $\mathrm{N}[\mathrm{C} @ \mathrm{H}](\mathrm{C}) \mathrm{C}(=\mathrm{O}) \mathrm{O}$. Thus, a complete representation of a component of a homogeneous mixture consists of a linear message identifying the component followed by a linear message giving the concentration as mole fraction such as $179482(-7)$, which is 0.0179482 (dimensionless). All of the components in a linear array with punctuation marks between them constitute a chemically defining message, and the structural complexity is the length of the symbol string. This is not yet the shortest message since Ziv-Lempel compression or some other compression may be used in reducing the message to a shorter form, but this method indicates an approach to the measure of complexity for biological solutions that might be extended to more elaborate structures. For regular polymers, considerable compression is possible. For a linear polymer such as a protein, structure is specified by sequence and Ramachandran angles. Per monomer, this requires one symbol of twenty and two numerical values to about three significant figures for most applications.

What the previous exercise makes clear is that we can in principle develop a metric for structural complexity in terms of the constructs of covalent bond chemistry, which is after all at the root of biochemistry. In principle, there is no reason why the method cannot be extended to other forms of bonding, in which case the fixity of the structures needs to be modified by essentially energetic and kinetic considerations. This will complicate the situation to a considerable degree

Bioevolution, in its most global aspects, is the transformation of the earth's chemistry from its prebiotic state, to cellular life, to the development of the rich biota and biogenic products represented by the many millions of species, present and past, and the fossil chemicals.

Chemical reactions that could be called prebiotic took place on earth about four billion years ago. The processes grew more complex, and they gave rise to life and the universal ancestor. That life has persisted and evolved with increasing complexity since that time. These statements stem from empirical generalizations in geochemistry, paleontology, biochemistry, and systematics and phylogeny. Every organism is a lineal descendent of the universal ancestor.

The purpose of this monograph is to provide a theoretical understanding of the development of biochemical net- works and subsequent biological complexity and evolutionary unfolding. This is done through postulating a scheme of nested shells of reaction networks of increasing complexity and sophistication. At the most reductionist and universal levels of biological chemistry these shells can be delineated with some precision, and the nature of the emergence by which new shells arise can be outlined in some detail. It has been noted [3] that biology must be understood at two levels: the physical level and the historical level. Study from the reductionist viewpoint involves traditional physical chemical approaches, while the historical perspective can be informed by the newer methods of complex adaptive systems.

$\mathbf{T}$ o expand on this, we assume that all biological processes at the prenoetic level, that is, before thought appeared, can be analyzed by and reduced to constructs of quantum mechanics, thermodynamics, and kinetic theory, as embodied in the periodic table and chemical bonding theory. However, even from a reductionist viewpoint, the evolution of biological systems may involve chance events and frozen accidents, rendering their history indeterminate in detail. To provide a framework for distinguishing historical aspects of biology from the more deterministic ones, consider the following abstraction: an ensemble of earths, a very large collection of identical planets in macroscopically identical solar systems 4.0 billion years ago before there were biological entities. Each of these earths will unfold in time and will have features that may be compared. There will be common properties that belong to the deterministic, domain, and distinctive features that are historical in nature. Each of these earths is an instantiation of the biologist's notion of "replaying the tape of evolution." It should be noted that the ensemble-of-solar-systems approach involves extending the Gibbsian ensemble construct of equilibrium statistical mechanics [4] into an entirely new domain far from equilibrium. It is presented for heuristic reasons only because we, at present, cannot treat such an ensemble analytically. We lack anything like the ergodic hypothesis that would make calculation possible.

The earth or each member of the ensemble 4.0 billion years ago had a surface that was a hot aqueous system with a steamy atmosphere. Occasional meteoritic impacts volatilized large quantities, or in some cases all, of the water. We assume the same impact profile for each ensemble member. The atomic composition of the surface had, more or less, the same value as the present one except for an unknown value for hydrogen, which constantly escapes from an aqueous planet of the size, density, and temperature of the Earth.

In this discussion, only the prenoetic domain of biology is under consideration. It seems clear that the emergence of the mind in animal evolution represents the appearance of radically new features, which will not be dealt with in this essay that focuses on the prehominid period. In any case the "gedanken" construct of the ensemble allows us to distin- 
guish the average features of the collection from the historical variation of the individual members.

Many of the aspects of life on earth follow in a necessary fashion from biota arising and existing in an aqueous environment. The properties of water [5], particularly in the liquid and solid states, mainly follows from the large dipole moment due to charge asymmetry along the oxygen hydrogen bond, the noncovalent hydrogen bonds, and the spatial distribution of electrons about the hydrogen and oxygen. All ensemble members of planets will have watery surfaces.

The theory we are developing is reductionist in that it depends on atomic properties outlined in the periodic table. Indeed, a major goal of a general biochemical theory of life is a mapping from the periodic chart of the elements onto the chart of metabolic pathways. The theory requires the introduction of constructs suggested by both physics and biology. These constructs are:

1. Far from equilibrium entity.

2. Replicating a far-from-equilibrium entity.

3. Postreplicative memory.

4. Competition for resources.

5. Structural complexity.

These constructs will now be defined in a more detailed manner.

1. Far from equilibrium entity:

a. An entity is defined as the contents of an imaginary closed surface in ordinary three-dimensional space.

b. A stable entity is an entity the contents of which remain associated over a period of time, during which they would normally dissociate by diffusion or other effects of thermal noise. Thus, the imaginary surface is replaced by a real barrier that restricts the free flow of molecules.

c. A far-from-equilibrium entity is an entity that, if placed in a rigid adiabatic container with walls coincident with the defining surface, will decay to a very different state (equilibrium), usually with a rise of temperature.

2. Replicating a far-from-equilibrium entity: This requires a stable far-from-equilibrium entity with a variable material bounding envelope, which takes in material from the outside. This envelope grows and divides the contents into two similar related entities that spatially separate. The material outside the original bounding element that enters the entity is designated as the nutrient and is part of the external environment.

3. Postreplicative memory: If a prereplication entity has certain properties that distinguish it from other such entities and its postreplication entities both have these properties to some degree, we refer to the process as having postreplicative memory.
4. Competition for resources: If two entities use common nutrients or energy sources, they are said to be in competition for resources. This usually applies to replication but may also apply to maintenance. Identifiable entities form a necessary basis for Darwinian competition.

5. Structural complexity: Complexity is the length of the shortest algorithm capable of generating a description of an entity at some point in time that is suitable for some predictive statement. The measure of complexity is thus dependent on the domain of the predictive statement, that is, the use for which the description will be applied, and the search for the shortest algorithm. One possible formalism has been discussed briefly earlier.

W ith the preceding definitions in mind, we note that a feature of life on earth as we know it is that it consists of stable replicating entities, separated from the homogeneous phase system and preserving individual integrity. This is particularly important because competition between all replicating entities, in a Darwinian sense, is a primary feature of life on earth. It presumably began as soon as identifiable, stable, self-replicating entities existed in the system.

Entities must exist and be distinguishable in order for Darwinian competition to occur. The notion that the particles of physics, such as electrons, are indistinguishable while the units of biology, such as cells, are complex and, therefore, distinguishable was one basis of a very challenging book by Walter Elsasser, The Physical Foundation of Biology [6]. He followed this up by three other monographs, Atoms and Organism [7], The Chief Abstractions of Biology [8], and Reflections On a Theory of Organisms [9]. In an age when molecular biology, with its firm reductionism, was making giant strides, Elsasser was widely misunderstood, but he introduced epistemological questions into the study of biology that are indeed profound.

He argued that while physics uses the epistemological universals of space, time, and causality, a fourth construct, "memory," must be introduced into biology. Current concepts of complexity and a deeper understanding of evolution going back into biogenesis serve to clarify some of Elsasser's ideas. As a physicist he was a confirmed reductionist who believed that nothing that ever happened in biology violated the laws of physical chemistry, which he took to be quantum mechanics. Nevertheless, he thought that there were biological laws that could not be derived from quantum mechanics.

Biological systems are a subset of all systems that have a common genealogy going back to the original ancestor some four billion years ago. What is surprising at first is that certain features have remained invariant over this history. This invariance defines what we include as biological systems. Complexity, replication, and competition are the operative constructs in biology. 
The basic unit of biology capable of replication with memory is the cell. Minimum cell size, both experimentally and theoretically, involves dimensions on the order of 0.1 to 0.28 micrometers $(\mu)$ or volumes on the order of $10^{-3} \mu^{3}$ to $8 \times 10^{-3} \mu^{3}$. Thus, there are on the order of $10^{9}$ atoms in a minimal cell. At the atomic level, no two cells can be identical; they are simply too complex. Using ideas that he traces back to Neils Bohr, Elsasser argued that such objects cannot be subject to exact quantum mechanical analysis. We cannot start with a number of objects all in the same state and then follow the statistics.

$\mathrm{t}$ is central to atomic physics that no two electrons or two protons are distinguishable. At the core of evolution is the idea that all organisms are distinguishable. Biological behavior is an emergent property at a certain level of complexity and phase separation. Each organism is a historical phenomenon carrying evidence of its four-billion-year-old past. In any case, complexity assures us that all the objects of biology (cells and organisms) are different.

Replication, the arising of offspring similar to the organisms from which they have come, assures us of a class of objects similar enough to use the notion of a class or species. If all offspring were identical, we could have no evolution. If all offspring were radically different, we could have no evolutionary continuity, no speciation. At some intermediate range, the evolutionary development of a planet is possible.

Competition organizes the selection among not identical offspring and provides the pruning algorithms for evolution. Thus, complexity, replication, and competition provide the basic driving forces for diversity but leave out the unity among diversity so characteristic of biology.

From the point of view of physical chemistry, being an entity requires phase separations in the sense implied in the Gibbs phase rule. In aqueous environments, the principle feature governing phase separation is electrical asymmetry, usually noted by such parameters as dialectic constant, electronegativity, or polarizability. The primary rule of phase separation under these circumstances is that oil and water do not mix. Oils are dominated by quite symmetric $\mathrm{C}-\mathrm{H}$ bonds and have dielectric constants below 10. Water is dominated by the electrically asymmetric $\mathrm{O}-\mathrm{H}$ bonds and has a dielectric constant of 80. It requires considerable energy to dissolve an oil molecule in water because to do so involves breaking many hydrogen bonds between water molecules. The primary features of phase separation lie deep within the physical features of small mobile electrons, large relatively immobile nuclei, the Pauli exclusion principle, and the distribution of electrons in covalent bonds. These universal physical features will, of course, emerge in all members of the ensemble of solar systems. They are predicted by quantum chemistry.

The necessity of being an entity is not required in those theories of biogenesis that are based on molecular sophis- tication developing in liquid phase environments. I would argue against this view on the grounds that increasing structural complexity is a property of organized replicating structures with memory, and it is not possible to have such structures without the prior existence of entities. The entities I am postulating are bounded by structures that form due to local free energy minima and will eventually decay to a global minimum if there is no flux of energy through the system. The kind of entities we are discussing do not have to be vesicular. They can exist as colloidal particles [10] or at phase interfaces [11].

A few features of thermal physics should be introduced at this point. If a nonequilibrium entity is adiabatically isolated, it will decay to an entropy maximum and will be characterized by maximum molecular disorder. The equilibrium state is always a global attractor leading to the breakdown of entities. To maintain a nonequilibrium entity requires as a necessary condition the flow-through of energy from an effectively high-energy (high-frequency) source to an effectively low-energy (low-frequency) sink [12].

There are two principle sources of energy flow in Earthlike planetary systems. The first is the flux of solar energy with a color temperature of the order of $5750^{\circ} \mathrm{K}$. The second is the release of magmatic reductants from beneath the crust. The leak of hydrogen from the planet leaves sufficient oxidants so that the planetary oxidants and reductants form couples, the reactions of which lead to the release of energy. These two sources seem to account for all biotic energy flux. The sink for both of these processes is the $3^{\circ} \mathrm{K}$ cold of outer space. The planet is constantly radiating infrared to this cosmic sink.

To think of a model of the equilibrium state to which a living system will decay, take some reference living system such as an organism, a terrarium, an aquarium, or any of the microcosms and mesocosms that have been studied and surround that system with a wall that will permit the flow of heat and is impermeable to the flow of matter and light. Place the isolated system in an isothermal reservoir at its initial temperature and allow it to age. It will slowly decay to equilibrium and maximum entropy following the second law of thermodynamics, which applies to such isolated systems. Dayhof and colleagues [13] have developed an algorithm to determine the chemical composition of a specified system of carbon, hydrogen, nitrogen, and oxygen atoms when it reaches the equilibrium state. This is done by minimizing the Gibbs free energy subject to the constraints.

The equilibrium systems are dominated by small molecules such as $\mathrm{CO}_{2}, \mathrm{H}_{2} \mathrm{O}, \mathrm{N}_{2}, \mathrm{NH}_{3}$, and so on. This decay toward equilibrium, which is a true global attractor, is also taking place in every living system but is countered by the anabolic processes, which are powered by the flow through of light to heat or of redox potential to heat. Note that the equilibrium state will be characterized by a well-defined measure of structural information by our previous criteria 
and that this measure will have a low value due to the small size and relatively small number of types of molecules that dominate at equilibrium.

Because of the global attractor nature of the equilibrium state, all the members of our ensembles of earths will have an appreciable concentration of the same small, energetically low-lying molecules. By energetically low lying, we mean a large negative energy of formation of the compound from its constitutive element (Gibbs' free energy of formation).

Returning to the problem of entity formation, among the variety of possible and likely covalent molecules in a nonoxidizing aqueous system containing carbon are the amphiphilic structures that have a polar portion and a nonpolar porture. An example of such a molecule is octadecanoic acid (stearic acid).

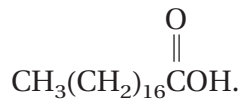

It possesses an alkane portion and a carboxylic acid that dissociates

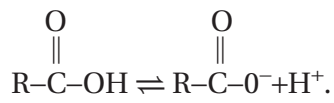

Thus, there is a highly polar charged end and a nonpolar alkane end represented by $R$. Because amphiphiles overwhelming concentrate at gas-water surfaces, they can have surface concentrations far in excess of their bulk-phase concentrations by several orders of magnitude.

Aniphiphilic molecules in water form a number of highly specific microphases, including various coacervates. Of special interest in biogenesis is a phase consisting of a planar biomolecular layer with the apolar portions in contact with each other and the polar portions in contact with the surrounding aqueous medium. Such structures can form into another microphase-vesicles-with an aqueous interior, a biomolecular leaflet membrance, and an aqueous exterior.

Consider next contemporary biological structures. There are three basic methods of structure building: macromolecules, membranes, and combinations of the two. The first two are composed of molecular subunits. In biological macromolecules these are the monomers that may form either precise or statistical polymers. Nucleic acids and proteins are precise polymers, and carbohydrates may exist in straight chain or branched polymers that do not have completely defined sequences, although there is a statistical regularity in nearest-neighbor frequencies.

In membranes the subunits analogous to monomers are the amphiphiles. The membrane structure forms as a local free energy minimum with only the most general of rules governing the composition in terms of the amphiphilic sub- units. This is because the subunits are not covalently bonded to each other. In short, there are two types of fundamental structures, each made of subunits in the molecular weight range of 80 to 600 daltons. In the first class of structures the sequences and other details are precisely defined as in the overall structure. In the second, the governing principle is less informational and more thermodynamic, so that a much greater degree of variation is permitted in the components and their relation to each other. Biological entities are predominantly bounded by the imprecise or sloppy variety or structures. We are also arguing that the membrane structure is likely to precede precise structures.

All contemporary cells are separated from their environments by plasma membranes, which have the same type of physical chemical structure as the simple bimolecular leaflet of amphiphilic molecules that we have been discussing. This membrane is responsible for the separation of organisms from the environment. It is the structure that makes the cell an entity and permits one to talk of the chemical composition of the cell's interior. It also allows for potential transmembrane differences, $\mathrm{pH}$ difference, and differences in oxidation reduction potentials. In short, much of what it takes to be a living cell is already present in amphiphillic vesicles.

Vesicle-forming molecules have been extracted from carbonaceous chondrites, can easily by synthesized by the solar irradiation of alkanes at oil-water interfaces, and in general can be expected in moderately reducing environments. Their synthesis belongs to this statistical order from the disorder domain of Schrödinger [14], and they could thus be expected in all or almost all members of the planetary ensemble we previously discussed.

$\mathbf{F}$ rom experimental studies, bilayer vesicles appear to have a preferred size in the range of 0.5 - to $50-\mu$ diameter, which is, of course, the preferred size range of present-day living cells. Surface forces can perhaps explain this size domain.

The advantages of vesicles as reactions vessels when compared to the open ocean is immediately apparent. A molecular event in the open ocean giving rise to a useful molecule will result in a concentration of about $10^{-45}$ moles/liter. A similar event inside a vesicle will result in a concentration of the order of $10^{-9}$ moles/liter. Nanomolar concentrations are clearly within the range of chemical interest where concentrations of $10^{-45}$ moles/liter are truly homeopathic.

Other types of entities are of course possible, including crystals, glasses, and coacervates. These do not lend themselves to the roles of reaction vessels and to the requirement of self-replication. Although coacervates have been suggested $[10,15]$, the fact that vesicles map over directly into the contemporary structure of biological cell plasma mem- 
branes is another argument for favoring them as the initial entities. The principle of continuity is an important methodological construct $[16,17]$. Other surfaces, such as clay and pyrite, have also been suggested, but they present problems in continuity.

Some self-replicating far-from-equilibrium entities can, in principle, act in the most general way by acquiring material from the environment and processing it to give rise to two or more similar entities, each with the ability to continue the process.

Associated with self-replicating far-from-equilibrium systems is an energy requirement. There is a constant thermal degradation, driven by the second law of thermodynamics, toward equilibrium that must be countered by the expenditure of work. Thus, there is the requirement of a proper energy source and the ability to convert that energy into a form that is useful in maintaining the structure and synthesizing similar structures.

In order for a vesicle entity to become a self-replicating entity, it must exhibit two features: the ability to synthesize membrane amphiphiles from substances in the environment and the ability to process available energy to develop an anabolism to counter the catabolic decay inherent in the thermal degradation, which, as has been noted earlier, is closely associated with the second law of thermodynamics.

There are, as we have noted, only two sources of energy continuously available on the terrestrial surface. The one most used in present-day biology is solar radiation with the approximate spectral distribution of a $5750^{\circ} \mathrm{K}$ source filtered by the atmosphere. Solar flux must be converted to the chemical free energy of a restricted set of well-defined compounds in order to be part of an anabolic process maintaining order. This places a number of restrictions on photosynthesis.

The second possible source of chemical energy consists of oxidation-reduction couples ultimately operating between reduced magmatic materials, which were brought to the surface by plate tectonics, and the oxidizing atmospheric and lithospheric components, which are usually the ultimate result of the decomposition of water and the leakage of hydrogen from the atmosphere. In a rather profound way, leakage of hydrogen at a fast rate compared to the leakage of oxygen is a significant feature of life on a planet where oxidation reduction reactions are an important feature of the biogeochemistry. In so far as these features are necessary for life on a planet, this places a limit on the fraction of possible biotic loci in the universe. Under sufficiently reducing conditions, $\mathrm{CO}_{2}$ can serve as an oxidant.

Other sources of energy are potentially available, such as the decay of long-lived isotopes, heat release due to shrinkage of the planet under gravity, and celestial mechanical features, such as tides. However, in general there are no ways of coupling these low-frequency sources to chemical potentials, so they need not, at this level, be considered from the point of view of biological organization.

In current biochemical processes two modes of energy processing predominate: group transfers involving the hydrolysis of phosphate ester bonds, and oxidation-reduction reactions. The two classes are themselves coupled at membranes chemo-osmotically (the Mitchell hypothesis), so there is great unity to all biotic energy processing. Restricting the chemical forms of energy flow in a reacting system restricts the possible chemical networks in that system. Thus, the universal features of energy processing limit the available biochemicals in an emerging metabolism. These are very significant constraints.

Given a group of complex, self-replicating, far-fromequilibrium entities, differences will arise between the entities due to chance occurrences. If these differences persist through the process of replication, the phenomenon of postreplication memory generates recognizable species of entities. In contemporary biology, memory is compartmentalized in the DNA genome, and species differences are by and large genetic differences. The phenomenon of memory need not always have been reified in such a precise and elaborate way. For example, some biologists have postulated an earlier state of evolution when RNA was both the message and the genetic structure. This requires considerably less molecular apparatus than the present structure. Memory can also exist without specific macromolecules but may be instantiated in chemical networks, catalytic loops, and reflexive autocatalysis. Any of these simple ways also leads to speciation, that is, to populations of distinguishable replicators with different chemical processes, efficiencies, and other physicochemical differences. Memory does not require macromolecules.

Replicators by their very nature must use environmental material and energy sources. The inherent finiteness of resources of necessity puts distinguishable species of replicators in competition with each other. Thus, all the Darwinian features of survival and fitness emerge from a situation with any kind of postreplicative memory and do not require a full genetic system.

Before attempting to develop the chemical network structure, we turn to the universal foundation on which our theory is based.

\section{ASSUMPTIONS}

O ne method of searching for the reactions of prebiotic chemistry is to start with the metabolic pathways chart from contemporary organisms and analyze the time sequence in which various steps must have come about. Several assumptions are involved in the present analysis:

1. The universal features in the contemporary metabolic pathways chart reflect the pathways of the original ancestor and are thus a virtual fossil of earliest life. They 
have been preserved by continuous self-replication and have retained their integrity because changes at the lowest level of a hierarchical and highly interconnected structures are almost guaranteed to be deleterious. Thus, the virtual fossil evidence extractable from the metabolic chart is older than the oldest extant rock on the surface of the Earth. The oldest rock from the Isua formation in Greenland is dated at 3.8 billion years.

2. The earliest ancestors were autotrophs, for heterotrophy imposes demands on the chemical composition of the environment for specificity and concentration that cannot be fulfilled by any known geochemical processes. All present-day heterotrophs rely on autotrophs to synthesize the precursor molecules. Both photoautotrophs and chemoautotrophs make all carbon compounds from carbon dioxide as the starting material. In trying to reconstruct prebiotic chemistry, we thus focus on autotrophic pathways. This is in line with the postulate that biogenesis proceeds from chemical simplicity to chemical complexity. We can hierarchically order compounds by counting how many chemical reaction steps they are from carbon dioxide. Therewill be an index number assignable to each carbon in a compound. Note that in highly reducing environments, carbon dioxide may be replaced by formic acid via the redox reaction.

$$
\mathrm{CO}_{2}+\mathrm{H}_{2} \rightleftharpoons \mathrm{I}-\stackrel{\mathrm{O}}{\|}-\mathrm{OH}
$$

3. At some early stage, phase separation was required. This may be archived in one of two general ways: (a) absorption on a surface or (b) trapping in a coacervate. For the former, suggestions have been made of clay-water interface, air-water interface, and pyrite-water interface. For the latter, oil droplets, proteinoids, and amphiphile vesicles have been suggested. Of all of these possibilities, only vesicles show a high degree of continuity with the universal cell structure. Vesicles require amphiphiles to be present at the outset in the environment. This does put some demands on the chemical composition of the environment. The requirement is nonspecific. Vesicles can be made from complex mixtures of amphiphiles. Only small amounts of such surface agents are required. In any case, very early in the biogenesis of the kinds of cells we know, a major task must have been the synthesis of amphiphiles. This will be true regardless of the earliest phase separations mechanism.

4. Biogenesis and the maintenance of life requires a continuous flow through of energy from high-frequency sources to low-frequency sinks. Among contemporary autotrophs, this is accomplished in two ways:

a. Photosynthesis, in which the source is solar photons and the ultimate sink is the $3^{\circ} \mathrm{K}$ cold of outer space; and b. Chemosynthesis with redox couples. This can be represented as

$$
\begin{aligned}
& \text { Reductant } 1+\text { Oxidant } 2 \rightleftharpoons \text { Reductant } 2 \text { - Oxidant } 1 \\
& \Delta \mathrm{G}<\mathrm{O} \\
& \mathrm{G} \text { is the Gibbs Free Energy }
\end{aligned}
$$

Here the high-frequency source is the energy released by the reaction. Most of the energy in the contemporary ecosphere comes from photosynthesis. For biogenesis, chemosynthesis is inherently simpler because it does not require the light-harvesting and energy-transducing components to convert photon energy into the redox couples that are required for cellular energy utilization. The sink is also the $3^{\circ} \mathrm{K}$ cold of space.

Energy in redox reactions is used as an analogy with frequency in photochemical reactions. The effective frequency $v_{\mathrm{e}}$ can be gotten from

$$
h v_{e}=\Delta G
$$

where $h$ is Planck's constant.

The difficulty in developing photosynthesis is not the absorption of photons, particularly in the high-frequency domain. The problem is to transduce the energy from the excited electronic state to a chemically useful form before it causes random chemical reactions that tend to be destructive. An example of this occurs in the photodynamic effect, in which visible energy absorbed by vital stains leads to cell death. The fact that there are only two classes of photosynthetic pigments in all of biology, chlorophylls and retinals, suggests that there may be something very special about an appropriate energy transduction.

5. At the prenoetic level, before thought appeared, biological processes can be analyzed and reduced to constructs of quantum mechanics, thermodynamics, and kinetic theory as embodied in the periodic table and chemical bonding theory. Biogenesis should be reduceable to the level of experimental laboratory chemistry.

6. The development of biological complexity, of which biogenesis is a part, can be analyzed through a hierarchy of nested reaction networks involving increasing complexity. Nests are, of course, hierarchical and represent the evolutionary course of biogenesis. At the core, the behavior tends to be governed by deterministic physical chemistry, and, as one moves out from the core, frozen accidents play an ever-increasing role in the historical unfolding of biology. As has been noted [3], biology stands between physics and history.

\section{METABOLIC PATHWAYS}

(2) ince the subsequent analysis is based predominantly D on an analysis of the metabolic pathways chart, we - will outline some of its general features. It is a vast empirical generalization based on a century and a half of 
work by an army of biochemists who have labored to characterize all of the chemical reactions taking place in living cells. It begins with the work of Justin Liebig, Claude Bernard, Louis Pasteur, and the Buchner brothers. At least 19 Nobel prizes have been awarded for work done in elaborating the chart. Despite all the honors bestowed on those who have developed parts of the structure, the entire chart is underappreciated as one of the great intellectual achievements of mankind. It is a case where the whole has a significance that goes far beyond the sum of the parts.

What makes the metabolic chart so significant is its universality and, therefore, the sense in which it then represents the metabolism of the universal ancestor. In the drawings of the scheme, the chart is presented as a graph in which the nodes are defined chemicals, such as pyruvate, glutamic acid, and cytosine, and the lines are chemical reactions, which connect the nodes in the following ways:

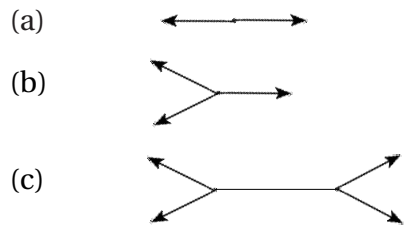

This is distinguished from ordinary graph theory in which all connections are of the class $a$.

The chart, as it is usually drawn in biochemistry texts, reflects both its historical origins and the stress on human metabolism and medicine that has been a driving force for the science of biochemistry. For our purposes, we shall make the distinction between primary and secondary metabolism. The former is that part of the chart that is shared by all autotrophs. Heterotrophs will be missing those portions of primary metabolism, the products of which are obtained from the environment by absorption or ingestion. Primary metabolism consists of those reactions that transduce energy; synthesize necessary monomers, cofactors, and other small molecules; and assemble the molecules of cellular functions: proteins, nucleic acids, carbohydrates, and lipids. The number of molecules; with relatively low molecular weights involved in the core metabolism is limited-on the order of a few hundred compounds. Primary metabolism cuts across all taxa and is clearly close to or at the evolutionary origin.

Secondary metabolism encompasses a vast number of taxon-specific reactions that have evolved along the tree of life and are the biochemical part of evolutionary radiation. An example of a secondary metabolite is cholesterol, which occurs only in the Animalia. Another example of secondary metabolism is involved in the excretion of nitrogen by animals. In the process of eating foods for energy, animals ingest far more nitrogen in protein than they require. This excess must be excreted. Marine animals tend to do this as ammonia. Most mammals excrete urea, and birds and rep- tiles tend to eliminate nitrogen in uric acid. These secondary pathways are clearly not required by plants and bacteria. We will confine our subsequent discussion to primary metabolism.

\section{THE SYNTHESIS OF MOLECULES IN AUTOTROPHS}

C entral to the metabolism of autotrophs is a set of reactions designated as the tricarboxylic acid cycle (also known as TCA cycle, Krebs cycle, or citric acid cycle). All major synthetic routes have a direct contact to the TCA cycle and an attached pathway from glycolysis. Thus, in autotrophs, amino acids are made from pyruvate, oxaloacetate, and $\alpha$-ketoglutarate; all lipids are made from acetate (in the form of acetyl coenzyme A [CoA]); and all sugars are made from phosphoglycerate. Thus, if we think of the TCA cycle and its immediate attachment as primordial, we have established the inner shell for biosynthesis (see Figure 1), with a carbon dioxide molecule being given off in the step to $\alpha$-ketoglurate and in the step to succinate.

\section{GETTING TO THE CORE OF THE METABOLIC CHART}

Since the initial stage of autotrophic metabolism functions to incorporate carbon from carbon dioxide into metabolic intermediates, we look at present-day carbon fixation to locate the core more exactly. Among the autotrophic bacteria there are three $\mathrm{CO}_{2}$ fixation pathways.

1. The major entry of $\mathrm{CO}_{2}$ into the present-day biosphere is by a reaction that may be simplified to

Ribulose 1,5 bisphosphate $+\mathrm{CO}_{2} \rightarrow$

2 molecules of 3 phosphoglycerate.

The energy to generate ribulose 1,5 bisphosphate comes in the form of ATPs and NADHs, which in photo-

FIGURE 1

Conventional TCA Cycle in Oxidative Mode

Acetyl CoA $\rightarrow$ Citrate
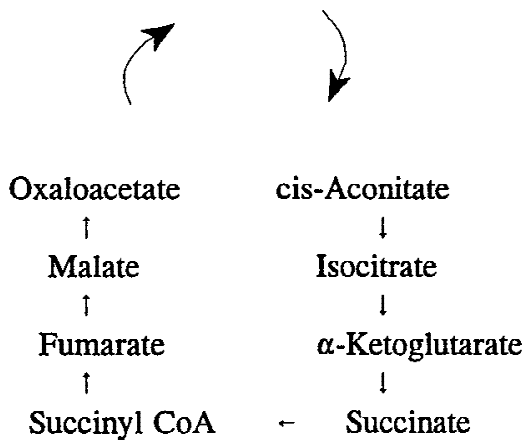
synthetic organisms are generated in the light reactions. The enzyme that catalyzes the reaction shown here is ribulose 1,5 bisphosphate carboxylate, which is also known as Rubisco.

Ribulose 1,5 bisphosphate is synthesized in the rather complex Calvin cycle, involving fructose 6 phosphate, glyceraldehyde 3 phosphate, xyulose 5 phosphate, erythrose 4 phosphate, dihydroxyacetone phosphate, sedoheptulose 7 phosphate, ribose 5 phosphate and the enzymes transketolase, aldolase, phosphatase, phosphopentose epimerase, and phosphoribulose kinase. Thus, $\mathrm{CO}_{2}$ incorporation through this pathway requires a complex set of enzymes as well as a source of pyrophosphate and reducing power as NADH or similar compounds. These can come from photosynthesis or from reactions of environmental oxidants and reductants for some lithoautotrophs. Rubisco is a dominant protein in most ecosystems.

2. A second family of $\mathrm{CO}_{2}$ fixation methods is referred to as the reductive acetyl CoA pathway. The reaction is characterized by one highly reductive pathway going to $\mathrm{CH}_{3-}$, while another $\mathrm{CO}_{2}$ goes to the carboxy acid level, and the two combine to form acetyl CoA. This system uses complex cofactors of the tetrahydrofolate system. Further $\mathrm{CO}_{2}$ incorporation takes place in going from acetyl CoA to pyruvate to oxaloacetate, either directly or through P-enolpyruvate.

3. A third pathway of total $\mathrm{CO}_{2}$ incorporation appears to be the simplest of all in terms of cofactors, chromophores, and other helper molecules. It has been designated the reductive citric acid cycle and has been demonstrated in Hydrogenobacter thermophilus, Hydrogenobacter acidophilus, Chlorobium limicola, and Desulfobacter hydrogenophilus. This cycle proceeds in the exact reverse of the usual citric acid cycle, with citrate being cleaved to acetyl CoA and oxaloacetate. The acetyl CoA then combines with $\mathrm{CO}_{2}$ to yield pyruvate. The pyruvate combines with $\mathrm{CO}_{2}$ and reductant to yield oxaloacetate. The entire scheme is represented in Figure 2.

This cycle has the following properties:

a. It is autocatalytic, since one molecule of citrate leads to the synthesis of two molecules of citrate.

b. The entire process involves only eleven kinds of molecules.

c. The reaction direction is determined by a strong reducing environment and by $\mathrm{CO}_{2}$ acting as an oxidant, leading to citrate and water.

\section{Reductive TCA Cycle}

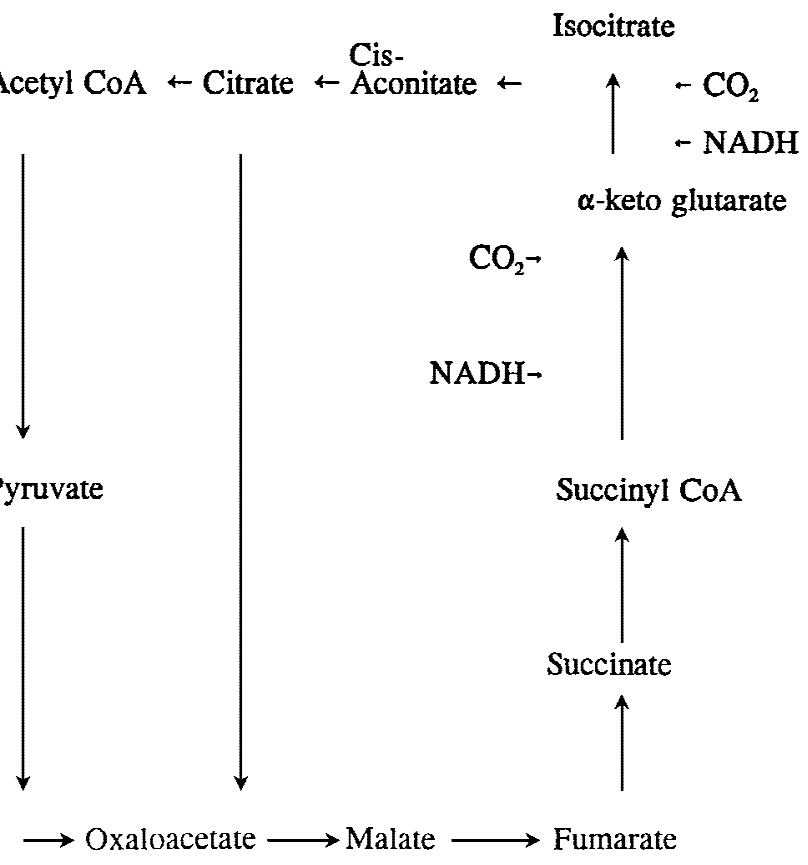


To make amphiphiles, the condensation reactions have to be followed by dehydration and reduction reactions. In the present-day biosphere, all of these reactions are enzymatically catalyzed. The extent to which they can proceed in the absence of enzymes is a question to be investigated in the laboratory.

$\mathbf{T}$ he next step is to look at amphiphile formation in present-day organisms. The general process of fatty acid synthesis proceeds as follows:

$$
\begin{gathered}
\text { acetyl ACP }+\mathrm{ATP}+\mathrm{CO}_{2} \rightarrow \text { malonyl } \mathrm{ACP}+\mathrm{ADP}+\mathrm{P} \\
\mathrm{C}_{2_{\mathrm{n}}} \text { acyl ACP }+ \text { malonyl ACP } \rightarrow \mathrm{C}_{2_{\mathrm{n}+} 2} \text { acyl ACP }+\mathrm{CO}_{2},
\end{gathered}
$$

where ACP stands for acyl carrier protein. The process has several features. In going from 2 to 4 carbons, the first product is acetoacetate, which is reduced to hydroxybutarate, dehydrated to crotonate, and reduced to butrate. The process proceeds by the addition of 2 carbon chains up to $\mathrm{C}_{16}$. The overall reaction is:

$$
\begin{aligned}
& 8 \text { acetyl CoA }+7 \mathrm{ATP}+14 \mathrm{NADPH} \\
& \quad \rightarrow \text { palmitate }+14 \mathrm{NADP}^{+}+8 \mathrm{CoA}+6 \mathrm{H}_{2} \mathrm{O}+7 \mathrm{ADP}+7 \mathrm{Pi}
\end{aligned}
$$

In any case, vesicles that can process energy and synthesize amphiphiles will clearly be self-replicating systems in the sense that we have discussed earlier. If they are in an environment with appropriate energy sources and sufficient numbers of low-molecular-weight precursors for the synthesis on amphiphiles, the membranes will grow and the vesicles will eventually divide.

A second postulate of the theory is that the evolution of self-reproducing systems proceeds by adding layers of complexity to the existing reaction network while maintaining the essential core structure. The best-understood instantiation of this idea can be seen in the reaction networks of intermediary metabolism. We will analyze these briefly in terms of shells of reactions in autrotrophs and will later return to a more detailed view. Because new functions accumulate at the periphery, we will further postulate that the intermediary metabolism of autotrophs recapitulates biogenesis.

\section{THE METABOLIC CHART: SHELLS A, B, C AND D}

The central shell of the metabolic chart consists of the citric acid cycle, the pathway from acetate to long-chain fatty acids and the pathway from acetate to glucose, which in the other direction is called glycolysis. Although numerous cofactors and enzymes are used at the substrate level, the shell consists of compounds of $\mathrm{CH}$ and $\mathrm{O}$. The role of phosphorus will be discussed later. Sulfur and thioesters appear as important intermediates even at the earliest time [19].
The inner core of metabolism is the citric acid cycle in which, under aerobic conditions, acetate is oxidized to two carbon dioxides with the concomitant reduction of three NAD and one FAD from the energy released. The reduced compounds are central in the energy metabolism via oxidative phosphorylation, and the molecules of the cycle are precursors to the synthesis of the essential compounds of cell growth, including amino acids.

The acetate in many species comes from pyruvate along the glycolytic pathway. The parallel pentose phosphate pathway may produce pyruvate, NADPH, or a variety of synthetic precursors. The reactions of photosynthesis feed into the existing pathways. Acetate is also used for the synthesis of fatty acids.

All of the reactions of the pathways listed here one feature in common. At the substrate level they exclusively involve compounds of carbon, hydrogen, oxygen, phosphorous, and sulfur. Since they process energy and lead to amphiphile synthesis, they are the sine qua non of the metabolism of the type of protocells previously discussed. At the substrate level there are no compounds in these pathways involving nitrogen, although this element plays a dominant role in the enzymes and cofactors (in particular coenzyme A) involved in the reaction sequences under discussion. We designate the network of reactions and substrates we have been discussing as shell A.

A functioning shell A synthesizes long-chain hydrocarbon precursors to amphiphiles and processes energy for synthetic reactions. The energy compounds need not be nitrogenous but might be polyphosphates and simple reductants such as formate and $\mathrm{H}_{2} \mathrm{~S}$ or $\mathrm{CH}_{3} \mathrm{SH}$. Thus, an appropriate shell A could drive a self-replicating entity. Hexose and pentose synthesis also belong to shell A. Note that shell A molecules alone lack amino acids, cofactors, and peptides. Shell A lacks any conventional biological enzymes. Hence, a pre-enzymatic (prebiotic) shell A would have to proceed without the usual catalytic functions as presently constituted. The extent to which this nonenzymatic chemistry is possible in aqueous media is an experimental question and is currently being investigated.

The entry of nitrogen into metabolic processes has certain features of interest. At present it enters the metabolism of the biosphere in the most reduced form, ammonia. It enters by a very small number of pathways with one pathway dominant, which involves glutamate and glutamine. This statement is of ecological as well as biochemical significance. Although nitrogen may enter the cells as atmospheric $\mathrm{N}_{2}$ or as various oxides, it is always reduced to $\mathrm{NH}_{3}$ before entering the synthetic pathways.

In the overall scheme of metabolism, shells are connected by gateways. In designating shells we only deal with the substrate-level molecules, not with cofactors and enzymes. 
The first shell then consists of glycolysis, the citric acid cycle, and the pathways of fatty acid synthesis. The gateway to the second shell (B) includes those reactions that convert keto acids to amino acids. The second shell encompasses most pathways to amino acid synthesis. The third shell (C) involves incorporating sulfur into the amino acids cysteine and methionine. The gateways to the fourth shell (D) involves ring closure and synthesis of nitrogen and dinitrogen heterocycles. The fourth shell consists of purines, pyrimidines, their derivatives, and a large number of cofactors. Most water-soluble vitamins are shell D molecules.

Other shells can be assigned in terms of sterol synthesis and diverse reaction families. The first shell is concerned with energetics, amphiphile synthesis, and precursors to biosynthesis. The second shell is concerned with amino acids and catalysis. It is the point where chirality becomes central in biosynthesis. The third shell provides the basis for more sophisticated protein structure. The fourth shell produces molecules to fine-tune reactions in the first two shells and template molecules leading to coding.

We now turn attention to the postulate that the order of biogenesis was shell A, shell B, shell C, shell D. We also opt for the simplest method of carbon fixation as the most probable earliest shell A. Here, as noted, the reductive tricarboxylic acid cycle, as demonstrated in a number of autotrophs, is the clear choice for simplicity.

\section{SUBSTRATES AND REACTIONS OF THE REDUCTIVE TCA}

\subsection{Substrates}

1. 2 carbon acetic acid or acetyl CoA

2. 3 carbon pyruvic acid

3. 4 carbon oxaloacetic acid

4. 4 carbon fumaric acid

5. 4 carbon malic acid

6. 4 carbon succinic acid or succinyl CoA

7. 5 carbon $\alpha$-keto glutarate (2oxoglutarate)

8. 6 carbon isocitric acid

9. 6 carbon cis-aconitate

10. 6 carbon citric acid

The study of biogenesis will focus on the possibility of the reductive cycle taking place without enzymes and serving as a precursor to biosynthesis. Some details of the reductive tricarboxylic acid cycle that was briefly outlined earlier are as follows:

1. Splitting reaction. Citric acid is cleaved to oxaloacetic acid and acetic acid. In the present enzymatic reaction, citrate plus ATP plus CoA yields acetyl CoA plus oxaloacetate plus ADP plus phosphate. The cleavage of ATP supplies energy for an endergonic reaction.

2. The minor loop goes from acetyl CoA to pyruvate incorporating a $\mathrm{CO}_{2}$. A second step condenses pyruvate and
$\mathrm{CO}_{2}$ to synthesize oxaloacetate. Thus, citrate goes to two oxaloacetates and ultimately to two citrates. Two $\mathrm{CO}_{2}$ molecules are incorporated in the minor loop.

3. The major loop from oxaloacetate to citrate proceeds by reduction to malate, dehydration to fumarate, reduction to succinate, then substitution to succinyl CoA, two reductive carboxylations to isocitrate, and rearrangement through cis-aconitate to citrate.

The reaction network has been diagrammed in Figure 2 . Let us consider further features of the network.

1. It is self-replicating. One complete cycle from citrate leads to two citrates.

2. It is an engine of synthesis:

acetate $\rightarrow$ lipids

pyruvate $\rightarrow$ carbohydrates

keto acids (oxaloacetate, $\alpha$-ketogularate, pyruvate) $\rightarrow$ amino acids

3. It allows complete mapping to the universal chart of intermediary metabolism.

4. Thermodynamics of the cycle. The overall cycle may be stoichimetrically represented as:

$$
9 \mathrm{H}_{2}+6 \mathrm{CO}_{2} \rightleftharpoons 1 \text { citric acid }+5 \mathrm{H}_{2} \mathrm{O}
$$

where the reducing equivalents are represented as coming from $\mathrm{H}_{2}$. Using Gibbs free energy values, the $\Delta \mathrm{G}$ of this reaction is

$$
\begin{aligned}
\Delta \mathrm{G}= & -42.54 \text { kjoules } / \text { mole citrate starting from aqueous } \\
& \mathrm{CO}_{2} \text {, and } \\
\Delta \mathrm{G}= & 10.68 \text { kjoules } / \text { mole citrate starting from gaseous } \mathrm{CO}_{2} .
\end{aligned}
$$

If we start with six of the reducing equivalents as formic acid, then we can write

$$
\begin{aligned}
& 3 \mathrm{H}_{2}+6 \mathrm{H}_{2} \mathrm{CO}_{2} \rightleftharpoons 1 \text { citric acid }+5 \mathrm{H}_{2} \mathrm{O} \\
& \Delta \mathrm{G}=-252.64 \text { kjoules/mole citrate }
\end{aligned}
$$

Next consider the reaction

$$
1 \text { citric acid } \rightleftharpoons 1 \text { acetic acid }+1 \text { oxaloacetic acid. }
$$

For this reaction,

$$
\Delta \mathrm{G}=2.31 \text { kilojoules } / \text { mole citrate }
$$

(The thermodynamic values are taken from the data summary of Miller and Smith-Magowan [20].)

The point to be made from these values is that in a grand canonical ensemble with $\mathrm{H}_{2}$ and $\mathrm{CO}_{2}$ reservoirs we would expect to find the citric acid cycle intermediates in high 
concentration. The starting materials for the core of the metabolic chart have a high probability in a moderately reductive $\mathrm{CO}_{2}$ world.

In a highly reductive world the following reaction takes over:

$$
4 \mathrm{H}_{2}+\mathrm{CO}_{2} \rightleftharpoons \mathrm{CH}_{4}+2 \mathrm{H}_{2} \mathrm{O}
$$

for which $\Delta \mathrm{G}=-132.77 \mathrm{kjoules} / \mathrm{mole}$ methane. The presence of $\mathrm{CO}_{2}$ would argue that the environment was not in such a reducing mode.

The problem now becomes experimental in that we can combine different ratios of $\mathrm{CO}_{2}$ and $\mathrm{H}_{2}$ in a thermal bomb, run the temperature up and down to allow some kind of chemical equilibrium to occur, and then determine the products. The calculations contain a strong suggestion that there is a reductive level at which citric acid cycle intermediates will dominate. The experiments are in progress.

\section{A SIMPLER SYSTEM}

rom the point of view of biogenesis we might inquire as to whether there is a simpler network that has the properties of the reductive TCA cycle. The following scheme is possible.

$$
\begin{aligned}
& \mathrm{H}_{2} \quad \mathrm{H}_{2} \\
& \downarrow \quad \downarrow \\
& \text { Acetate } \leftarrow \text { Succinate } \leftarrow \text { Fumarate } \quad \text { Succinate yields } \\
& \begin{array}{rlrl} 
& \downarrow & \uparrow & \text { two acetates } \\
\mathrm{CO}_{2} \rightarrow & \downarrow & \uparrow & \\
2\left[\mathrm{H}_{2}\right] \rightarrow & \downarrow & & \uparrow \rightarrow \mathrm{H}_{2} \mathrm{O} \\
& \downarrow \mathrm{CO}_{2} & 2\left[\mathrm{H}_{2}\right] & \uparrow \\
& \downarrow & \downarrow & \downarrow
\end{array}
\end{aligned}
$$

The pathway from oxaloacetate to succinate involves the reduction.<smiles>O=C(O)C(C(=O)O)C(=O)O</smiles>

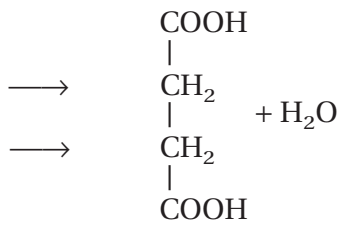

These are a set of simple reactions leaving the essential as

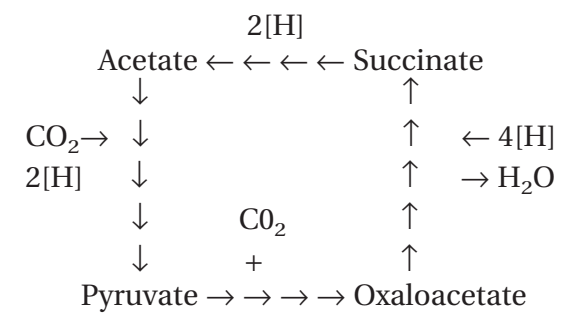

Succinate yields two acetates
The reactions for the simplified pathway are:

A. the reductive carboxylation of acetate to pyruvate;

B. the carboxylation of pyruvate to oxaloacetate; and

C. the reductive steps leading from oxaloacetate to succinate and water. This may involve several steps.

$\mathbf{T}$ he essential role of sulfur is open to discussion. Although the substrates of the reductive cycle are not structurally dependent on sulfur, they nevertheless use acetyl CoA and succinyl CoA with the thiol linkage. In a prebiotic stage these might be replaced by thioacetate and thiosuccinate. Just as polyphosphate might play the role of ATP in the prebiotic world, reduced sulfur compounds such as $\mathrm{H}_{2} \mathrm{~S}$ and $\mathrm{H}_{3} \mathrm{CSH}$ might play the role of reduced CoA. Wächterhauser [11] has written extensively on the possible role of sulfur compounds in biogenesis, and the focus on the reductive TCA cycle resonates with some of his ideas. deDuve [19] has made a strong case for the role of thiol esters. In any case, the redox couples of the prebiotic shell A may well involve sulfhydryl groups.

The route of nitrogen in present-day autotrophs starting from various redox states always leads to ammonia, which is then incorporated by two reactions. In the first of these, anunonia and $\alpha$-ketoglutarate condense to form Lglutamate. NADPH serves as the reductant in this reaction. In the second reaction, glutamate and ammonia form glutamine. This is driven by the hydrolysis of ATP. All subsequent aminations are amino transfer reactions starting from the $\alpha$-amino group of glutamate or from the secondary amino group of glutamine. The first of these reactions has been carried out nonenzmatically [21].

The second shell consists of the reactions and substrates starting from the first shell by the addition of amino nitrogens and subsequent rearrangements. The second or B shell includes most of the amino acids. Just as the A shell is the energetic and amphiphilic shell, so the B shell is the catalytic shell because the primary path to catalysis is by the formation of peptides leading eventually to long-chain catalytic peptides or enzymes. Emerging from the second shell are found a set of sulfur fixation reactions. Plants and most microorganisms utilize the reaction.

$$
0 \text { - acetylserine }+\mathrm{H}_{2} \mathrm{~S} \rightarrow \text { cysteine }+ \text { acetate }+\mathrm{H}_{2} \mathrm{O}
$$

Other microorganisms can utilize serine directly

$$
\text { serine }+\mathrm{H}_{2} \mathrm{~S} \rightarrow \text { cysteine }+\mathrm{H}_{2} \mathrm{O}
$$

All other sulfur compounds in the ecosystem are synthesized with cysteine as the primary sulfur source. Because of the importance of S-S linkages in cysteine and methionine 
as chain initiators, the third shell sulfurs play a major role in determining protein configurations.

Note that the second shell is dependent on the first. Amino acids are synthesized from a series of compounds starting with $\alpha$-ketoglutarate, oxoloacetate, 3 phosphoglycerate, pyruvate, phosphoenolpyruvate, erythrose 4 phosphate, and ribose 5 phosphate. These are all shell A compounds.

A relatively restricted gateway connects shells $\mathrm{A}$ and $\mathrm{B}$, and it drives the fixation of $\mathrm{NH}_{3}$ in the biosphere. It is a crucial step toward the existence of shell $\mathrm{B}$.

The addition of sulfur to the amino acids depends on a functional shell A and shell B and should be regarded as the gateway to shell $\mathrm{C}$ that allows for a more sophisticated set of peptides, including cysteine and methionine. Since cysteine plays a unique role in the tertiary structure of proteins, and since methionine plays a unique terminal role in amino acid sequence, the fact that shell $\mathrm{C}$ involves only a small number of compounds should not detract from the significance of this shell in leading to structural sophistication of macromolecules. The shell $\mathrm{C}$ sulfur compounds are also on the way to certain vital shell D cofactors, such as CoA. The oxidation reduction reactions in the sulfhydryl groups play major roles in contemporary biochemistry. The entry of sulfur into amino acids depends on a small number of gateway reactions from shell B to shell $\mathrm{C}$.

Shells $\mathrm{B}$ and $\mathrm{C}$ result from the incorporation of new elements, nitrogen and sulfur, into the metabolic network in an informational way, although sulfhydryl groups may have been used in an energetic way in the original shell A. The D shell involves a different type of innovative behavior. The gateway consists of new synthetic pathways leading to nitrogen and dinitrogen heterocycles. Again, a restrictive number of gateway reactions from A, B, C, and D shells results in a variety of biochemical behaviors that radically alter the behavior of the emerging system. Consider the shell D compounds. They include the nucleic acid, purines and pyrimidines, and most of the water-soluble coenzymes. The development of the system of templates allows a totally new way of storing and transmitting information. A different use of information is also seen in the ability of coenzymes to fine-tune shell A, B, C, and D reactions.

Many of the vitamins, including thiamine, riboflavin, folic acid, and vitamin $B_{12}$, are shell D compounds. The difficulty of synthesis of these molecules may result in loss of a pathway when the substance is present in available foods. Although it is not at present totally clear whether mono nitrogen heterocycles, such as nicotinic acid and vitamin $B_{6}$, are shell D or shell B compounds, we shall tentatively regard them as shell D compounds.

Shell D is synthesized from shell A, B, and C molecules. A principle characteristic of present-day shell D is the ubiquity of dinitrogen hetreocycles. Purines are synthesized on ribose phosphate (shell A) and consist of components con- tributed by glutamine, glycine, aspartate, $\mathrm{CO}_{2}$, and formate. Inositate, the first purine in the pathway, can then be converted to adenylate and guanylate. Pyrimidines are made from glutamine, $\mathrm{CO}_{2}$, and aspartate. The first product is orotate. Orotate is then combined with a ribose phosphate and further nucleotides are formed.

The existence of shell D as noted leads to two classes of molecules: the template-active monomers of RNA and DNA, and the cofactors such as ATP, NAD, NADP, FAD, and CoA.

To move from these results, which come from extending the metabolic chart to a more general theory, assume that self-replicating vesicles competed and some shell A reactions proved to have a high degree of fitness. Some events led to the gating reactions that resulted in shell $\mathrm{B}$. Those vesicles in which shell B molecules were catalytic for shell A reactions were more fit and out-competed the others. A series of shell B and shell A reactions led to shell C. Shell A, $B$, and $C$ reagents are the precursors of shell D. And so the system proceeds.

This analysis in terms of shells may have more general biological applicability than intermediary metabolism and relates to the question of why evolution leads to increasing complexity of chemical reaction networks, of cells, of organisms, and of ecosystems. To focus on this we recall Dollo's Law, an evolutionary principle that was formulated by the Belgium paleontologist Louis Dollo in the mid-1890s.

Dollo asserted that evolution was irreversible, that one could not trace back the very complicated series of steps to recover an existing structure. For example, when the precetaceans, land mammals that evolved into whales, returned to the sea, they possessed embryonic pharangeal arches, which in their evolutionary precursors, the fish, led to gill slits for breathing in water. They could not retrace the evolutionary path to gill slits but instead developed a series of new structures to add to the mammalian lung-based respiratory system. This allowed them to function for long periods under water. This irreversibility stems from the fact that biological systems are both complex and highly interrelated. To trace back stepwise a path that once went forward, without selection along the way, seems so highly improbable as to be ruled out.

B ecause of the complexity and connectedness, random changes other than those near the periphery of the network in the outer shells have an enormously high probability of upsetting the system and having a negative fitness value. As a result, beneficial changes can occur most easily by adding at the edges of the network. By network, we designate a chemical reaction set or any functional set of interactions at a higher level of organization. As a result, networks grow more and more complex. These are gradual changes and allow for a slow evolution. At some point a gateway transition takes place that opens up a previously unavailable pathway leading to major changes of the sys- 
tem. Such a gateway step leads to an explosive radiation. An example we have discussed is the gateway reaction from $\alpha$-ketoglutaric acid to glutamic acid to glutamine. Evolution then proceeds by steady gradual additions followed by episodic gateway events that open up new shells of structure. This is a chemical statement of the idea of punctuated equilibrium. Chemically, the gateway events may be outlined with some precision.

The availability of a novel shell of processes or structures allows for new functions, some of which have a fitness advantage. The opening up of gateways constitute, as noted, punctuation events in the current rhetoric of evolution. At the level of this discussion, the gateway events are new syntheses giving rise to chemicals with novel properties.

Consider the following possible gateway events: (1) the conversion from ribose to deoxyribose nucleotide, which allows the transfer of genetic storage from RNA to the more stable DNA and (2) the synthesis of cholesterol and related sterols, which permits stable cells without walls that can engulf particles by endocytosis. This opens the possibility of cells eating other cells (animalness) and the development of organelles by endosymbiosis (eukaryoteness).

The theory under consideration, which is rooted in intermediary metabolism, seems to have a more general embodiment. Consider entities with the following properties:

1. Memory: Each generation of entities acts as a pattern (template) for the next generation.

2. Competition: Each entity competes with similar and different entities for environmental resources.

3. Interactive complexity: Each entity consists of a number of highly interconnected subunits.

4. Mutability: Some subunits or connections can undergo random change.

5. Shell structure: Networks of subunits have hierarchical depth within the entities.

The properties we have just enumerated clearly characterize living organisms. In a competitive situation, survival depends on being able to mutate in response to changing environment. We conjecture that because of high connectivity, hierarchically deep mutations are likely to decrease fitness. They may help some property and harm some others. Thus, the core of this system will tend to stay the same while adding steps at the edges. Thus, there is a tendency for such systems to add small steps and to grow more complex. A gateway mutation opens up a whole new space of exploration with the possibility of increasing fitness while not interfering with the existing hierarchies. Thus, evolutionary radiations are likely to follow gateway events. Gateway mutations involve a level of innovation considerably higher than normal mutations. This all can be seen in the metabolic examples that have been discussed. Competition,

\section{FIGURE 3}

The energy amphiphile shell

$\downarrow \rightarrow \mathrm{NH}_{2}$ gateway

The amino acid shell

$\downarrow \rightarrow$ Incorporation of S into amino acids gateway

The protein shell

$\downarrow \rightarrow$ The dinitrogen heterocycle gateway

Information molecules

$\downarrow \rightarrow$ The deoxy gateway

DNA

$\downarrow \rightarrow$ The wall gateway

The universal ancestor

$\downarrow$

Prokaryotes
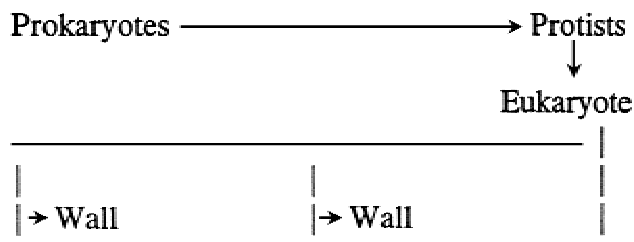

Plants Saprophytes Animals

complexity, and connectedness lead to more complexity. Figure 3 presents a large-scale model of this approach.

Let us proceed to a more conventional view of biological organization starting from the hierarchy shown in Figure 4.

FIGURE 4 
It is my contention that the organization of shells and gateway events characterize the entire development of complexity outlined here, although there may be branching in the hierarchy giving rise to an organizational tree such as that shown in Figure 3. The view is entirely reductionist in that each level may be studied in terms of lower levels, but it also admits emergence in that the gateway event gives rise to structures and/or processes that did not exist or to function in shells interior to the shell generated by the gateway event. It is not necessary to choose between reductionism and emergence.
The lower two levels on the hierarchy occur in the domain of atomic physics and chemistry. Because of limitations imposed by quantum mechanics and the structure of the periodic table, events at these levels occur within a limited space of possibilities. They should be repeatable in a chemistry laboratory and represent the part of the complexity problem that can be most easily reduced to experiment. At the lowest level we are concerned here with emergences in the ensemble of earths, not the more general problem of the emergence of complexity in some specific system.

\section{REFERENCES}

1. Zurek, W. Complexity $1995,1,64$

2. Weininger, D. J Chem Inf Comput Sci 1988, 28, 31-36.

3. Smith, T.; Morowitz, H.J. J Mol Evol 1982, 18, 265-282.

4. Gibbs, J.W. Elementary Principles of Statistical Mechanics; Longman Greens, London: 1902 (reprinted by Ox Bow Press: Woodbridge, CT, 1989).

5. Edsall, J.T.; Wyman, J. Biophysical Chemistry; Academic Press: New York, 1958.

6. Elsasser, W. The Physical Foundations of Biology; Pergamon Press: Elmsford, NY, 1958.

7. Elsasser, W. Atom and Organism; Princeton University Press: Princeton, NJ, 1966.

8. Elsasser, W. The Chief Abstractions of Biology; North-Holland Publishing Co.: New York, 1975.

9. Elsasser, W. Reflections on a Theory of Organisms; Editions Orbis Publishing: Toronoto, 1987.

10. Fox, S.W.; Nakashima, T. Biochim Biophys Acta 1967, 140, 155-167.

11. Wächterhäuser, G. Microbiol Rev 1988, 52, 452-484.

12. Morowitz, H.J. Energy Flow in Biology; Academic Press: New York, 1968 (reprinted by Ox Bow Press: Woodbridge, CT, 1979).

13. Dayhoff, M.; Lippincott, E.R.; Eck, R.V. Science 1964, 146, 1461.

14. Schrödinger, E. What is Life? Cambridge University Press: Cambridge, UK, 1945.

15. Oparin, A.L. The Origin of Life on Earth; Oliver and Boyd: Edinburgh, Scotland, 1957.

16. Morowitz, H.J. The Beginnings of Cellular Life; Yale University Press: New Haven, CT, 1992.

17. Fry, I. Biol Philos 1995, 10, 389-417.

18. Morowitz, H.J.; Heinz, B.; Deamer, O. Origins of Life and Evolution of the Biosphere 18, The Chemical Logic of a Minimum Protocell; 1988.

19. deDuve, C.V. Vital Dust; Basic Books, New York, 1995.

20. Miller, S.; Smith-Magowan, D. J Phys Chem Ref Data 1990, 19, 1049-1072.

21. Morowitz, H.J.; Peterson, E.; Chang, S. Origins of Life and Evolution of the Biosphere 25; 1995; 395-399. 